ARTÍCULO ORIGINAL

\title{
Consecuencias reproductivas y sanitarias de los abortos bovinos en la cuenca lechera del Valle de Lerma, Salta, Argentina
}

\author{
Suarez $\mathrm{VH}^{*}$, Martínez GM
}

Estación Experimental Agropecuaria Salta, Instituto Nacional de Tecnología Agropecuaria, Argentina.

* Correspondencia: Víctor H. Suarez, EEA Salta, INTA, Ruta Nacional 68 km 172 (CP: 4403) Cerrillos, Salta, Argentina. E-mail: suarez.victor@inta.gob.ar

Recibido: 22 Marzo 2021. Aceptado: 5 Agosto 2021. Disponible en línea: 6 Septiembre 2021

Editor: P. Beldomenico

\begin{abstract}
RESUMEN. Con el objetivo de investigar los efectos de los abortos bovinos en la cuenca lechera del Valle de Lerma se estudiaron los registros de datos de 128 vacas Holstein en ordeño obtenidos de software de 4 tambos. Los abortos se clasificaron en: vacas abortadas, vueltas a inseminar que continúan en lactancia (VAC, $n=36$ ) y vacas que abortaron e iniciaron en una nueva lactancia (VAl, $n=33)$, además se les sumaron los registros de vacas con partos normales $(V P N, n=59)$. Se registraron los litros de leche a los 305 días de lactancia (L/305), los días en lactancia (DEL), los intervalos entre partos (IPP), número de eventos de salud ocurridos pos aborto y el destino final de los animales (continúa en el tambo, descarte, muerte o sacrificio). Las variables continuas se sometieron a análisis de varianza en un modelo lineal mixto y las discretas a la prueba de Chi cuadrado y Odds Ratio (OR). No se hallaron diferencias entre los litros de leche producidos (L/305) por las vacas VPN $(8640$ I) y las VAC $(8498$ I) pero si $(p<0,0001)$ entre éstas y el grupo VAI $(5742)$, considerando las vacas de diferente número de lactancias y el tiempo gestacional del aborto. El grupo VAC tuvo DEL $(p<0,0001)$ e IPP $(\mathrm{p}<0,0001)$ respectivamente más prolongados $(639,7$ e 441,2 d.) que las vacas del VAl $(419,9$ e 404,2 d.) y VPN $(405,5$ e 476,7 d.). Las vacas abortadas (VAC + VAl) tuvieron una mayor $(\mathrm{p}<0,0001)$ probabilidad de contraer respectivamente desórdenes sanitarios y reproductivos (OR=6,5, IC 3,2 - 24,3) y descartes más muertes/sacrificios (OR=6,72, IC 2,9-15,1) en comparación con VPN. Estos resultados muestran los graves efectos de los abortos sobre la performance productiva y la salud de las vacas lecheras.
\end{abstract}

SUMMARY. Reproductive and health consequences of bovine abortions in the dairy basin of the Valle de Lerma, Salta, Argentina. In order to investigate the effects of bovine abortions in the dairy basin of Valle de Lerma records of 128 Holstein milking cows from the software of 4 dairy farms were studied. The abortions were classified into: aborted cows re-inseminated that continue in lactation (VAC, $n=36$ ) and aborted cow that started a new lactation (VAI, $n=33$ ) and were added the records of: cows with normal parturitions (NPV, $n=59$ ). Milk litters accumulated at 305 lactation days (L / 305), days in lactation (DEL), the intervals between calvings (IPP), number of post-abortion health disorders and number of cows that continues, culled, death or slaughtered were recorded. Continuous variables were subjected to analysis of variance with a mixed linear model and the discrete ones to Chi square test and Odds Ratio (OR). No differences were found between the milk litters production (L / 305) of NPV (8640) and VAC (8498) cows but there were differences $(p<0.0001)$ between those groups and the VAl group $(5742)$ considering the cows of different number of lactations and gestational time to abortion. The VAC group had DEL $(p<0.0001)$ and PPI $(p<0.0001)$ respectively longer $(639.7$ and 441.2 d.) than those of VAI ( 419.9 and 404.2 d.) and NPV (405.5 e 476.7 d.) cows. Aborted cows (VAC + VAl) had a higher $(p<0.0001)$ probability of contracting, respectively reproductive health disorders $(\mathrm{OR}=6.5, \mathrm{Cl} 3.2-24.3)$ and culls plus deaths / slaughters $(\mathrm{OR}=$ $6.72, \mathrm{Cl}$ 2.9-15.1) than those of NPV. These results show the serious effects of abortions on milk production, reproductive performance and health of dairy cows.

Palabras clave: aborto; bovino lechero; sanidad ganadera; Argentina

Keywords: abortion; dairy cattle; herd health; Argentina

\section{Introducción}

La sustentabilidad de las empresas dedicadas a la producción de leche depende en gran medida del desempeño productivo de las vacas, que está ligado al gerenciamiento y al manejo general de la empresa, basados estos en una correcta alimentación, una buena genética y un adecuado manejo reproductivo y sanitario. Dentro de estos factores un deficiente desem- peño reproductivo se traducirá en pérdidas económicas de consideración tanto en rentabilidad como en el progreso genético (Plaizier et al., 1997; De Vries, 2006).

Específicamente, una mala performance en reproducción puede deberse a una deficiencia en la detección de celos, problemas asociados a la fertilidad o pérdidas durante la gestación (Melendez y Pinedo, 2007). Un importante factor condicionante del buen desempeño reproductivo, productivo y sanitario de las vacas 
lecheras es el aborto. Estudios previos indican sus efectos sobre los rodeos al disminuir su productividad, incrementar los problemas sanitarios y el descarte de animales, reducir el número de vaquillonas de reemplazo y elevar los costos en alimentación, reproducción y tratamientos (Thurmond et al., 1990, Gädicke et al. 2010).

El aborto es en general definido como la muerte fetal ocurrida entre los 42 y 260 días de preñez (Peter, 2000). Si este ocurre antes del día 42 desde la concepción la pérdida se define como muerte embrionaria (Fetrow et al., 1990, Thurmond et al., 1990, Forar et al., 1996). Mientras que, aquellas pérdidas ocurridas con posterioridad al día 260 hasta el término de la gestación son denominados partos prematuros, ya que el ternero estaría en condiciones de sobrevivir (Miller, 1986; Gädicke y Monti, 2008).

La cuenca lechera del Valle de Lerma, se caracteriza por tener aproximadamente unas 35 empresas tamberas que en su mayoría tienen un manejo de base pastoril con fuerte suplementación, mientras que unas pocas han implementado sistemas más intensificados como "dry lots" y "free stalls". La producción promedio diaria es de $21,5 \pm 5,3 \mathrm{l} / \mathrm{vaca}$, mientras que la producción promedio anual por tambo es de $4900 \mathrm{l} /$ día (Suarez y Martínez, 2015). A pesar de que se trata de una cuenca en crecimiento tanto tecnológico como productivo, tiene muchos factores limitantes tanto en el aspecto reproductivo como sanitario, con tasas promedios de mortalidad o sacrificio y de descartes involuntarios de vacas respectivamente de $13,0 \pm 4,1 \%$ y de $12,3 \pm 5,2 \%$. Estos datos de mortalidad comparados con los de EEUU, Nueva Zelanda o los países de la Unión Europea son muy altos y muy poco competitivos (Thomsen y Houe, 2006).

A través de una encuesta llevada a cabo en tambos del Valle de Lerma en Salta, se registró en promedio una tasa de abortos del 7,8 $\pm 4,2 \%$ (Suarez y Martínez, 2015). Este número de abortos declarado seguramente estaría subestimado si se consideraran ciertas fallas de detección, como en aquellos casos donde el aborto de vacas dadas como preñadas que vuelven a celar no es registrado. A pesar de que un porcentaje de abortos del 3 al $4 \%$ es considerado aceptable (Weaver y Goodger, 1987; Peter, 2000), estudios en otros países muestran que la tasa de abortos en los tambos varía entre el 8 y $14 \%$ dependiendo del momento de la gestación en el cual la preñez fue diagnosticada y de los biotipos que componen el rodeo lechero (Thurmond et al., 2005; Norman et al., 2012; El-Tarabany, 2015; Catalino et al., 2017).

A pesar de las pérdidas económicas que ocasionan los abortos, hay muy poca información a nivel de las principales cuencas lecheras del país sobre su incidencia y efectos en los rodeos lecheros. Solo como antecedente y basados en informes de grupos CREA, Sancor y otras empresas lácteas en Santa Fe del año 2012, el porcentaje de abortos rondaría el 10\% (Abdala com. personal). A partir de esta carencia de investigaciones el presente estudio tiene como propósito describir los efectos tanto reproductivos como productivos de los abortos en las vacas de aquellos tambos con registros en la cuenca lecheras del Valle de Lerma.

\section{Materiales y Métodos}

\section{Ubicación y características de los tambos}

El estudio fue realizado en la cuenca lechera del Valle de Lerma, ubicada a 1050 y 1300 msnm, que comprende los departamentos Capital, Cerrillos, Rosario de Lerma y El Carril de la provincia de Salta. Las vacas en ordeñe de todos los tambos estudiados eran de raza Holstein representativas de los animales explotados en esta cuenca lechera.

Los tambos estudiados ( $n=4)$ fueron aquellos que contaban con software y registros de datos confiables. Tres manejaban al rodeo en base a pasturas y verdeos con un fuerte aporte $(75 \%$ en promedio de los requerimientos alimenticios) de suplementación. EI tambo restante manejaba su rodeo en total confinamiento en un sistema "free stall". En los 4 tambos se practicaban dos ordeñes diarios, los servicios eran continuos y el diagnóstico de preñez se realizaba mediante tacto rectal o ecografía (ultrasonografía) a los 35 -55 días pos inseminación.

\section{Registro de los datos}

Los datos utilizados en el estudio fueron relevados de los softwares de gestión de las empresas (Programa de gestión "DIRSA") de los 4 tambos visitados que tenían más de cinco años de registros de información.

A partir del diagnóstico de preñez, la confirmación del aborto se realizaba a partir de cualquier indicación de muerte fetal (hallazgo del feto, placenta o anexos embrionarios expulsados) o de la presentación de un nuevo celo en la vaca. El aborto (pérdidas fetales entre los 42 y 260 días de gestación) de 69 vacas fue dividido por sus características en dos grupos: a) vacas abortadas, vueltas a inseminar que continúan sin cambios en la presente lactancia (VAC; $n=36$ ), b) vacas que abortaron e iniciaron en una nueva lactancia (VAl; $\mathrm{n}=33$ ). No hubo diferenciación entre los abortos en función de si fueron por causas infecciosas o no infecciosas. También se registró esta información en un lote similar en cuanto a edad y número de lactancias de vacas que no padecieron un evento de aborto, es decir con partos normales (VPN; $n=59$ ).

A partir de estas bases de datos, en aquellas vacas que habían padecido un aborto se registraron con posterioridad al evento indicadores de eficiencia productiva, reproductiva y de salud comparándolos con lactancias de vacas sin abortos. Como índices productivos se registraron la producción de litros de leche acumulados 
a los 305 días de lactancia (L/305), la duración de la lactancia en días en lactancia (DEL). Como indicador reproductivo se registraron los intervalos entre partos (IPP) (Fetrow et al., 1990).

Como indicadores sanitarios se registraron el número de eventos de salud ocurridos con posterioridad al aborto y el destino final o no de la vaca, ya sea que continúa en el tambo, fue descartada involuntariamente y murió o fue sacrificada. Los eventos sanitarios considerados fueron solo los problemas reproductivos posteriores a los abortos registrados en el software. Estos fueron endometritis, retención de placenta, aborto, mastitis clínicas e infertilidad (vacas que no se preñan). Para el análisis de los descartes involuntarios (aquellos no referidos a la edad) y muertes posteriores al evento del aborto no se diferenciaron los eventos reproductivos del resto (afecciones podales, traumatismos, etc.), debido a la falta de precisiones en los diagnósticos de los tamberos y a la escasez de diagnósticos veterinarios; considerando que los sesgos eventuales estarían incluidos azarosamente en los tres grupos.

\section{Análisis estadísticos}

Para analizar diferencias entre variables continuas como L/305, DEL y IPP entre los grupos VAC, VAI y VPN se utilizó el análisis de varianza con en un modelo lineal mixto con el número de lactancias como efecto fijo, la edad gestacional del aborto como covarianza y su interacción. Inicialmente se realizaron para las variables la prueba de normalidad de Shapiro Wilk. Las diferencias entre las variables discretas se analizaron mediante la prueba de Chi cuadrado y para las asociaciones entre variables se utilizó el Odds Ratio (OR). Para todos los análisis estadísticos se consideró un valor de $\mathrm{P}<0,05$ como significativo utilizando el programa estadístico InfoStat, 2017.

\section{Resultados}

Se analizó en total el desempeño de 128 vacas de entre 19 y 42 animales por cada establecimiento lechero. La tasa de abortos en los cuatro tambos se ubicó entre el 7,5 y $9 \%$.

No se hallaron diferencias $(p<0,49)$ en los días de preñez al momento de abortar entre las vacas de $1 \mathrm{er}$ (162,3 $\pm 55,8$ días), 2 da (183,1 $\pm 54,5$ días) y 3er o más lactancias $(172,8 \pm 52,4$ días $)$, por el contrario se encontraron diferencias $(p<0,019)$ entre las VAC (aborto a los $156,1 \pm 48,9$ días) y las VAl (aborto a los $186,7 \pm$ 55,1 días), con un $33,3 \%$ y $66,7 \%$ respectivamente para VAC y VAI de abortos por sobre los 200 días de preñez. La Figura 1 indica el número de animales que presentaron abortos de menos de 100 , de 101 a 200 y de más de 200 días de preñez, indicando el bajo número registrado de abortos menores a los 100 días.

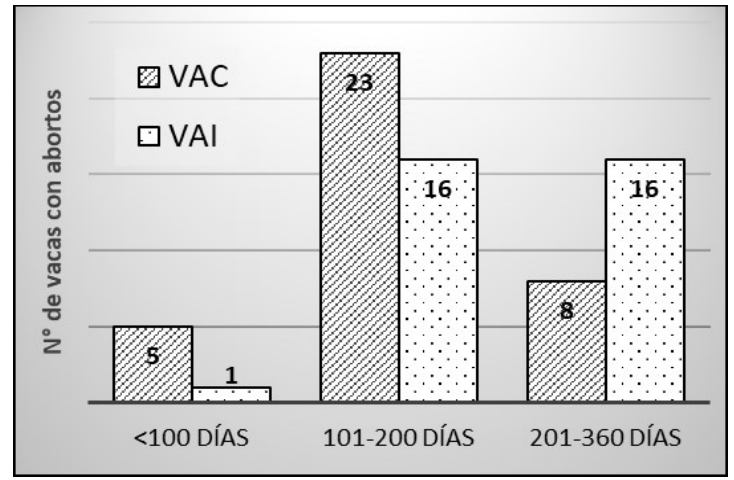

Figura 1. Número de vacas que presentaron abortos en el 1er, 2do y 3er tercio de la preñez de las vacas abortadas que continúan la lactancia (VAC) y de las que inician una nueva lactancia (VAI).

Los resultados relativos a la producción de leche L/305, DEL y IPP para las vacas totales de cada grupo y diferenciados en animales de 1ra, 2da y 3ra o más lactancias se incluyen en la Tabla 1 . No se hallaron diferencias significativas $(p<0,19)$ entre los animales de 1 ra, 2 da y de 3 o más lactancias en los rindes totales $\mathrm{L} / 305$, aunque los de primera lactancia tendieron a ser más bajos. No se hallaron diferencias entre la producción de leche $(L / 305)$ entre las vacas VPN y las VAC pero si $(p<0,0001)$ entre éstas y el grupo VAl, considerando las vacas de diferentes número de lactancias y el momento del aborto. Las vacas del VAC y VAI produjeron en promedio respectivamente 142,1 $(1,6 \%)$ y $3120,5(36,1 \%)$ litros menos que los que produjeron las vacas del VPN. Al clasificar las vacas entre aquellas por debajo y sobre los 8000 litros L/305 días se observa que las del VAl presentan una probabilidad significativa $(p<0,0001)$ de producir por debajo de esa cifra 65 veces mayor (OR 65,6; IC 6,9439) en comparación con las vacas que tuvieron partos normales (Tabla 2).

En cuanto al DEL, entre las vacas con diferente número de lactancias no hubo diferencias, pero si $(p<0,001)$ entre las vacas de los grupos VAC, VAl y VPN, presentando las del grupo VAC lactancias significativamente $(p<0,0001)$ más prolongadas. Los resultados de análisis de los DEL fueron para las vacas del VAC y VAl respectivamente de más 204,3 días $(48,9 \%)$ y 2,9 días $(0,7 \%)$ en comparación con las vacas con partos normales. Las vacas del VAC presentan una probabilidad significativa $(p<0,0008) 9,6$ veces mayor (OR 9,65; IC 2,1-44,2) de tener lactancias por sobre los 350 días que las vacas que tuvieron partos normales (Tabla 2).

En el IPP tampoco hubo diferencias entre las vacas con diferente número de lactancias, pero si $(p<0,0001)$ entre las vacas de los grupos VAC, VAl y VPN, presentando las del grupo VAC intervalos más prolongados. Los resultados IPP fueron de más 265,1 días $(55,4 \%)$ y de menos 72,5 días $(15,2 \%)$ para las vacas del VAC y VAI respectivamente, en comparación con las vacas del VPN. Al clasificar las vacas entre 
aquellas por debajo y sobre un IPP de 400 días se observa que las del VAC presentan una probabilidad significativa $(p<0,003) 13$ veces mayor (OR 13,5; IC 1,7 $108)$ de tenerlo por sobre esa cifra en comparación con las vacas que tuvieron partos normales (Tabla 2).

No se hallaron diferencias significativas entre las vacas de $1 \mathrm{ra}, 2 \mathrm{da}$ y $3 e r$ lactancia de los tres grupos en total en cuanto a la ocurrencia de eventos sanitarios (X2 1,28; $p<0,52)$ y de descartes más muertes ( $X 23,07 ; p<0,21)$, aunque como puede verse en la Figura 2, la de 1er parto tienden a tener un menor número de eventos $y$ un mayor número de descartes más muertes.

El número de eventos sanitarios registrados luego de los abortos en los tres grupos se señalan en la Tabla 3. El porcentaje de disturbios sanitarios relacionados con la reproducción fueron respectivamente para los grupos VAC, VAl y VPN del $53,3,51,9$ y $14,9 \%$ mostrando diferencias significativas (Tabla 4). La ocurrencia de eventos sanitarios de las vacas abortadas (VAC + VAI) mostró que estas tuvieron un mayor $(p<0,0001)$ riesgo de contraer enfermedades reproductivas en comparación con las que tuvieron partos normales $(\mathrm{OR}=6,5, \mathrm{IC}$ $3,2-24,3)$

Tabla 1. Producción media y desvíos estándar de litros de leche estimados a 305 días de lactancia (L/305), duración de la lactancia en días en lactancia (DEL) e intervalo entre partos (IPP) discriminados por el número de lactancias y la media total de las vacas abortadas que continúan la lactancia (VAC), las vacas abortadas que inician una nueva lactancia (VAI) y vacas con partos normales (VPN).

\begin{tabular}{|c|c|c|c|c|}
\hline Variables & $\begin{array}{c}\mathbf{N}^{\circ} \text { de } \\
\text { lactancias }\end{array}$ & VAC & VAI & VPN \\
\hline \multirow[t]{4}{*}{$L / 305$} & 1ra lactancia & $\begin{array}{l}7820 \\
\pm 1445\end{array}$ & $\begin{array}{l}5974,8 \\
\pm 1370\end{array}$ & $\begin{array}{r}7234,1 \\
\pm 1380\end{array}$ \\
\hline & 2da lactancia & $\begin{array}{r}8538,2 \\
\pm 1029\end{array}$ & $\begin{array}{l}5960,1 \\
\pm 2010\end{array}$ & $\begin{array}{l}8975,8 \\
\pm 1763\end{array}$ \\
\hline & $\geq 3$ ra lactancia & $\begin{array}{l}8935,9 \\
\pm 1472\end{array}$ & $\begin{array}{l}5343,4 \\
\pm 2214\end{array}$ & $\begin{array}{l}9156,5 \\
\pm 1887\end{array}$ \\
\hline & Total & $\begin{array}{r}8498 \\
\pm 1350 b\end{array}$ & $\begin{array}{c}5742,1 \\
\pm 1992 a\end{array}$ & $\begin{array}{r}8640,5 \\
\pm 1923 b\end{array}$ \\
\hline \multirow[t]{4}{*}{ DEL } & 1ra lactancia & $\begin{array}{c}672 \\
\pm 200\end{array}$ & $\begin{array}{l}507,6 \\
\pm 268\end{array}$ & $\begin{array}{l}421,4 \\
\pm 110\end{array}$ \\
\hline & 2da lactancia & $\begin{array}{c}708 \\
\pm 194\end{array}$ & $\begin{array}{c}389 \\
\pm 135\end{array}$ & $\begin{array}{l}431,2 \\
\pm 109\end{array}$ \\
\hline & $\geq 3$ ra lactancia & $\begin{array}{l}601,4 \\
\pm 198\end{array}$ & $\begin{array}{l}335,7 \\
\pm 217\end{array}$ & $\begin{array}{l}405,5 \\
\pm 128\end{array}$ \\
\hline & Total & $\begin{array}{c}639,7 \\
\pm 221 a\end{array}$ & $\begin{array}{r}419,9 \\
\pm 234 b\end{array}$ & $\begin{array}{r}417,0 \\
\pm 115 b\end{array}$ \\
\hline \multirow[t]{4}{*}{ IPP } & 1ra lactancia & $\begin{array}{c}706 \\
\pm 122\end{array}$ & $\begin{array}{c}415,5 \\
\pm 58\end{array}$ & $\begin{array}{c}436,3 \\
\pm 63\end{array}$ \\
\hline & 2da lactancia & $\begin{array}{c}796 \\
\pm 120\end{array}$ & $\begin{array}{c}399,5 \\
\pm 72\end{array}$ & $\begin{array}{l}491,4 \\
\pm 129\end{array}$ \\
\hline & $\geq 3$ ra lactancia & $\begin{array}{l}751,4 \\
\pm 160\end{array}$ & $\begin{array}{c}397,7 \\
\pm 60\end{array}$ & $\begin{array}{l}477,5 \\
\pm 153\end{array}$ \\
\hline & Total & $\begin{array}{r}741,2 \\
\pm 131 a\end{array}$ & $\begin{array}{r}404,2 \\
\pm 85 b\end{array}$ & $\begin{array}{r}476,7 \\
\pm 133 b\end{array}$ \\
\hline
\end{tabular}

Tabla 2. Odds Ratio, intervalos de confianza y valor $\mathrm{p}$ de presentar una producción leche estimados a 305 días de lactancia (L/305) mayor o menor de $8000 \mathrm{I}$, una duración de la lactancia en días (DEL) mayor o menor de 350 días y un intervalo entre partos (IPP) mayor o menor de 400 días entre las vacas abortadas que continúan la lactancia (VAC) y las vacas abortadas que inician una nueva lactancia (VAI) comparadas separadamente con aquellas con partos normales (VPN).

\begin{tabular}{|c|c|c|c|c|c|c|}
\hline \multicolumn{3}{|c|}{ Variable } & \multirow[t]{2}{*}{ OR } & \multirow[t]{2}{*}{ LI 95\% } & \multirow[t]{2}{*}{ LS 95\% } & \multirow[t]{2}{*}{ valor $p$} \\
\hline$L / 305 d$ & $\leq 80001$ & $>80001$ & & & & \\
\hline VAC & 11 & 25 & \multirow[t]{2}{*}{0,9} & \multirow[t]{2}{*}{1,69} & \multirow[t]{2}{*}{7,72} & \multirow[t]{2}{*}{0,860} \\
\hline VPN & 19 & 39 & & & & \\
\hline VAI & 32 & 1 & \multirow[t]{2}{*}{65,6} & \multirow[t]{2}{*}{6,9} & \multirow[t]{2}{*}{439} & \multirow[t]{2}{*}{$<0,001$} \\
\hline VPN & 19 & 39 & & & & \\
\hline DEL & $\geq 350 \mathrm{~d}$ & $<350 d$ & & & & \\
\hline VAC & 34 & 2 & \multirow[t]{2}{*}{9,65} & \multirow[t]{2}{*}{2,1} & \multirow[t]{2}{*}{44,2} & \multirow[t]{2}{*}{$<0,001$} \\
\hline VPN & 37 & 21 & & & & \\
\hline VAI & 16 & 17 & \multirow[t]{2}{*}{0,53} & \multirow[t]{2}{*}{0,22} & \multirow[t]{2}{*}{1,2} & \multirow[t]{2}{*}{0,150} \\
\hline VPN & 37 & 21 & & & & \\
\hline IPP & $\geq 400 \mathrm{~d}$ & $<400 d$ & & & & \\
\hline VAC & 27 & 1 & \multirow[t]{2}{*}{13,5} & \multirow[t]{2}{*}{1,7} & \multirow[t]{2}{*}{108,1} & \multirow[t]{2}{*}{0,003} \\
\hline$\overline{\text { VPN }}$ & 32 & 16 & & & & \\
\hline VAI & 7 & 9 & \multirow{2}{*}{$\begin{array}{c}0,3 \\
9\end{array}$} & \multirow[t]{2}{*}{0,12} & \multirow[t]{2}{*}{1,23} & \multirow[t]{2}{*}{0,11} \\
\hline VPN & 32 & 16 & & & & \\
\hline
\end{tabular}

Tabla 2. Eventos sanitarios relacionados con la reproducción posteriores a los abortos $\mathrm{o}$ a los partos normales en las vacas abortadas que continúan la lactancia (VAC), las vacas abortadas que inician una nueva lactancia (VAl) y las vacas con partos normales (VPN).

\begin{tabular}{|c|c|c|c|c|}
\hline \multicolumn{2}{|r|}{ Grupos } & VAC & VAI & VPN \\
\hline \multirow{7}{*}{$\begin{array}{c}\text { Eventos } \\
\text { posteriores }\end{array}$} & $\mathrm{N}$ & 19 & 31 & 47 \\
\hline & Endometritis & 3 & 6 & 4 \\
\hline & Distocia & 0 & 0 & 1 \\
\hline & Retención de placenta & 2 & 3 & 0 \\
\hline & Aborto & 4 & 7 & 1 \\
\hline & Mastitis & 0 & 2 & 1 \\
\hline & total & 9 & 18 & 7 \\
\hline \multirow{4}{*}{$\begin{array}{l}\text { Descartes } \\
\text { y muertes }\end{array}$} & $\mathrm{N}$ & 36 & 33 & 59 \\
\hline & Descartes involuntarios & 10 & 15 & 3 \\
\hline & Muertes/sacrificios & 8 & 7 & 10 \\
\hline & Total & 18 & 22 & 13 \\
\hline
\end{tabular}




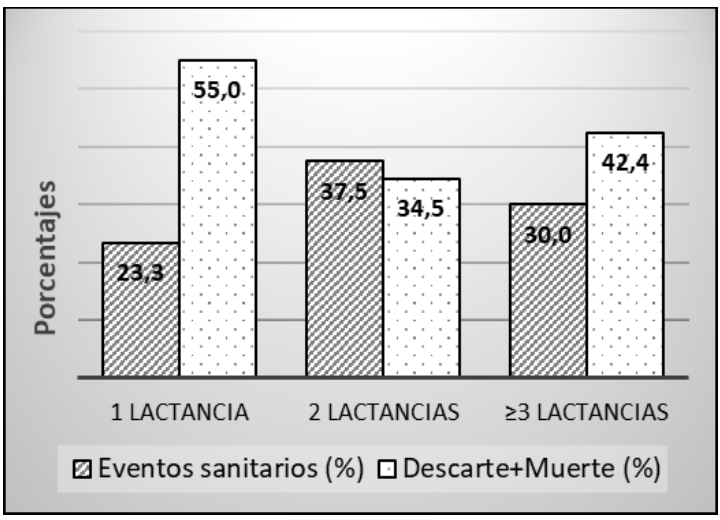

Figura 2. Porcentaje de eventos sanitarios reproductivos y descartes más muertes/sacrificio del total de animales de 1ra, 2 da y $\geq 3$ lactancias estudiados.

El número de eventos sanitarios registrados luego de los abortos en los tres grupos se señalan en la Tabla 3. El porcentaje de disturbios sanitarios relacionados con la reproducción fueron respectivamente para los grupos VAC, VAl y VPN del 53,3, 51,9 y $14,9 \%$ mostrando diferencias significativas (Tabla 4). La ocurrencia de eventos sanitarios de las vacas abortadas (VAC + VAl) mostró que estas tuvieron un mayor $(p<0,0001)$ riesgo de contraer enfermedades reproductivas en comparación con las que tuvieron partos normales $(O R=6,5$, IC $3,2-24,3)$.

El número de vacas descartadas y de muertas o sacrificadas de los 3 grupos se indican en la Tabla 3. El porcentaje de vacas descartadas más las muertas fueron respectivamente para los grupos VAC, VAI y VPN del $56,7,75,0$ y $22,0 \%$ mostrando diferencias significativas (Tabla 4). La ocurrencia de eventos sanitarios de las vacas abortadas (VAC + VAI) mostró que estas tuvieron un mayor $(p<0,0001)$ riesgo de ser descartadas o morir o ser sacrificadas que aquellas con partos normales (OR= 6,72, IC 2,9 - 15,1). La Tabla 4 muestra los OR, es decir la probabilidad de ocurrencia de eventos sanitarios y los descartes más las muertes/sacrificios entre las VAC y las VAI en comparación con las VPN. Estos resultados muestran que a partir de los abortos no solo se pierden terneros, sino que tienen consecuencias sobre la performance productiva y sanitaria.

\section{Discusión}

Los resultados del presente estudio, aunque referidos a las consecuencias de los abortos en tan solo cuatro tambos, tienen importancia debido a la falta de investigaciones en este tema en nuestro país. En cuanto a la presentación de los abortos investigados, la figura 1 muestra que los registros indican pocas vacas abortadas con menos de 100 días de preñez. Probablemente esto esté indicando dificultades por parte de los encargados de hallar abortos pequeños, que pasan inadvertidos. Forar et al. (1996) y Peter (2000) informaron que la detección de abortos a través de la localización del feto o las membranas fetales expulsadas solo sería del 20 al $45 \%$ y que este porcentaje se incrementaría al aumentar edad gestacional al momento del aborto. Además, es probable que la tasa de abortos registrada en los tambos esté subestimada debido a que según datos previos el mayor riesgo de pérdida fetal es durante el primer trimestre de gestación y luego progresivamente disminuye a medida que avanza la gestación con un ligero aumento del riesgo hacia el último mes de gestación (Thurmond et al., 1990; Wiltbank et al., 2016).

Tabla 4. Odds Ratio, intervalos de confianza y valor $\mathrm{p}$ del número de eventos sanitarios y descartes más muertes/sacrificio ( $D+M)$ entre las vacas abortadas que continúan la lactancia (VAC) y las vacas abortadas que inician una nueva lactancia (VAI) comparadas separadamente con aquellas con partos normales (VPN).

\begin{tabular}{|c|c|c|c|c|c|c|}
\hline \multicolumn{3}{|l|}{ Variable } & \multirow[t]{2}{*}{ OR } & \multirow[t]{2}{*}{ 니 95\% } & \multirow[t]{2}{*}{ LS 95\% } & \multirow[t]{2}{*}{ valor $\mathbf{p}$} \\
\hline $\mathbf{N}^{\circ}$ de eventos & con eventos & sin eventos & & & & \\
\hline VAC & 9 & 17 & \multirow[t]{2}{*}{3,02} & \multirow[t]{2}{*}{0,96} & \multirow[t]{2}{*}{9,4} & \multirow[t]{2}{*}{$<0,051$} \\
\hline VPN & 7 & 40 & & & & \\
\hline VAI & 12 & 9 & \multirow[t]{2}{*}{7,6} & \multirow[t]{2}{*}{2,3} & \multirow[t]{2}{*}{24,7} & \multirow[t]{2}{*}{$<0,0004$} \\
\hline VPN & 7 & 40 & & & & \\
\hline $\mathrm{N}^{\circ}$ de abortos posteriores & con abortos & sin abortos & & & & \\
\hline VAC & 4 & 16 & \multirow[t]{2}{*}{11,5} & \multirow[t]{2}{*}{1,19} & \multirow[t]{2}{*}{110} & \multirow[t]{2}{*}{$<0,025$} \\
\hline VPN & 1 & 46 & & & & \\
\hline VAI & 7 & 7 & \multirow[t]{2}{*}{46} & \multirow[t]{2}{*}{4,8} & \multirow[t]{2}{*}{432} & \multirow[t]{2}{*}{$<0,0001$} \\
\hline VPN & 1 & 46 & & & & \\
\hline $\mathrm{N}^{\circ}$ de descarte + muertes & con $\mathrm{D}+\mathrm{M}$ & $\sin \mathrm{D}+\mathrm{M}$ & & & & \\
\hline VAC & 22 & 14 & \multirow[t]{2}{*}{5,56} & \multirow[t]{2}{*}{2,23} & \multirow[t]{2}{*}{13,8} & \multirow[t]{2}{*}{$<0,0002$} \\
\hline VPN & 13 & 46 & & & & \\
\hline VAI & 22 & 11 & \multirow[t]{2}{*}{7,07} & \multirow[t]{2}{*}{2,7} & \multirow[t]{2}{*}{18,3} & \multirow[t]{2}{*}{$<0,0001$} \\
\hline VPN & 13 & 46 & & & & \\
\hline
\end{tabular}


A pesar de que diversos autores (Thurmond et al., 2005; Lee y Kim 2007; Ghavi Hossein-Zadeh, 2013) informaron que la probabilidad de aborto aumenta con el aumento de la edad en la concepción, con número creciente de abortos previos, y si en la preñez anterior al aborto fue de más de 60 días de gestación, los presentes resultados no evidencian diferencias entre vacas con diferente número de pariciones. También Thurmond et al. (2005) informa que la probabilidad de abortar disminuye a medida que el número de días abiertos aumenta, y que sería debido a que la involución uterina tendría más tiempo para recuperarse.

Los presentes hallazgos evidencian que el aborto está asociado a una baja en la producción de litros de leche fijados a $\mathbf{3 0 5}$ días, especialmente en vacas clasificadas como que inician una nueva lactancia. El aborto al inicio de una nueva lactancia asociado a una menor producción láctea podría deberse a la falta de desarrollo de las glándulas mamarias debido a un inicio temprano de la lactancia en comparación con lo que ocurriría en vacas con partos normales, donde el mayor desarrollo de estas se produce en los últimos 35 días preparto (Capuco et al., 1997; Wilde y Knight, 1998).

Estudios previos también demuestran un rendimiento productivo inferior con posterioridad a los abortos y partos prematuros (El-Tarabany, 2015, Bartels et al., 2006; Gädicke et al., 2010). Un trabajo realizado en Irán sobre vacas Holstein demostró que las vacas con partos normales tenían un rinde fijado a 305 días superior, además de un mayor porcentaje de grasa y proteínas en leche que las vacas que padecieron abortos (Ghavi Hossein-Zadeh y Ardalan 2011; Keshavarzi et al., 2020). Bartels et al. (2006), también informaron una disminución diaria de $0,72 \mathrm{~kg}$ de leche durante los primeros 100 días de lactancia en la lactancia pos aborto en vacas lecheras holandesas.

En cuanto al largo de los DEL, Keshavarzi et al. (2020) obtuvieron resultados similares a los del presente ensayo, observando que las vacas abortadas que continuaban en lactancia tuvieron una proporción mayor por sobre los 330 días en lactancia que aquellas vacas sin abortos, y que las vacas abortadas que iniciaban una nueva lactancia presentaban un número inferior en DEL que aquellas con partos normales.

Las variables reproductivas se vieron marcadamente afectadas por el tipo de aborto, con un menor y mayor IPP para las vacas clasificadas con VAl y VAC respectivamente en comparación con las de parto normal. Cabe aclarar que la clasificación y registros de si una vaca que aborta continúa la lactancia y se intenta servirla al primer celo o si inicia una nueva lactancia, sería una decisión del encargado o el veterinario de acuerdo a lo avanzado de la gestación al momento de la pérdida, la presentación o no de celo y el nivel de leche producida. Esta clasificación un tanto arbitraria seguramente estaría influyendo en los resultados, como por ejemplo un menor IPP de las VAl, las cuales poste- riormente a la pérdida fetal estarían mostrando celo. La inseminación de estas vacas sin considerar un periodo de espera de 45 días, favorecidos por la falta de un pico importante de producción de leche asociado a un menor estrés como las que tendrían un parto normal, podría explicar un periodo abierto más corto y un menor IPP en las vacas VAI. Se observó que el tiempo transcurrido de gestación al aborto influye sobre los días abiertos, aumentando el intervalo medio entre la pérdida fetal y la concepción en 54-63 días en el primer tercio de preñez, 85 días en el segundo y 102-116 días en tercer tercio de preñez (Lee y Kim 2007; Ghavi Hossein-Zadeh 2013).

Con respecto al efecto del aborto sobre los días abiertos y consecuentemente en gran medida sobre el IPP, analizando partos prematuros y abortos en forma general, El-Tarabany (2015) halló que vacas Holstein tuvieron IPP y días abiertos más largos después de haber sufrido partos prematuros (475 y 198 días) y abortos (427 y 151 días) que las vacas con partos normales (381 y 149 días). Sin embargo, los resultados del número de inseminaciones para lograr la concepción en las vacas con pérdidas fetales han sido controversiales: El-Tarabany (2015) halló un aumento en el número de inseminaciones (NI) por parto de 2,3 en las vacas abortadas ( $\mathrm{NI}=5,3)$ con respecto a aquellas con partos normales $(\mathrm{Nl}=3,2)$, mientras que por el contrario Bartels et al. (2006) informaron que el aborto no tuvo efecto sobre ninguna de las variables reproductivas como por ejemplo el número de inseminaciones por concepción. Pero los informes de Keshavarzi et al. (2020), quienes diferenciaron como en este estudio las vacas con abortos que continuaban la lactancia de aquellas que la iniciaban, hallaron un incremento de 1,6 servicios por preñez respectivamente para las primeras y un menor número $(0,17)$ de servicios para las que iniciaban lactancia al compararlas con las vacas de partos normales.

Una de las razones por las que las vacas clasificadas con VAl tendrían IPP algo menores y un número de servicios menor por preñez, obedecería a que estas vacas abortadas presentarían un balance energético negativo menor que aquellas que terminan la preñez normalmente, ya que es sabido que este balance negativo de las vacas lecheras propicia subfertilidad (Butler y Smith, 1989; Nigussie, 2018).

Dentro de los estudios sobre efectos económicos de los abortos, Rafati et al. (2010) informa que los intervalos entre partos de más de 425 días producirán pérdidas de más del $10 \%$ en la producción media de los tambos.

El presente trabajo, también evidenció un importante efecto de los abortos sobre la salud y el bienestar de las vacas, a partir de una probabilidad mayor de padecer eventos sanitarios reproductivos posteriores a la pérdida fetal, observándose que las inflamaciones uterinas y los abortos posteriores serían una de las patologías con mayor incidencia (Tabla 3). Las metritis en la 
producción lechera son de gran importancia principalmente porque aumentan el intervalo parto concepción y disminuyen la producción láctea (Fourichon et al., 2000). Estudios previos demostraron que aquellas vacas abortadas tenían mayor riesgo de presentar metritis acompañadas de sus efectos indirectos sobre la salud general y la producción de leche (Kaneene y Miller, 1995); en el mismo sentido Salasel et al. (2010) concluyeron que los partos anormales, incluyendo los abortos, pueden estar asociados con una mayor prevalencia de endometritis subclínicas como lo indicaría el aumento de neutrófilos en las muestras de lavado uterino. Los estudios de Gröhn et al. (1990) estimaron que las vacas que abortan tienen $19,8,3,7$ y 2,1 veces más probabilidad de tener respectivamente retención de placenta, metritis y celo silente. Otros estudios señalaron que las endometritis serían más frecuentes cuando las pérdidas durante la gestación ocurrirían en el tercer trimestre en comparación con el primer y segundo trimestre, con una prevalencia general del 23,2\% de endometritis (Lee y Kim 2007).

También el porcentaje de abortos posteriores a la pérdida fetal de la presente investigación fue un $22 \%$ superior al compararlo con los ocurridos en las VPN $(2,2 \%)$. Los análisis predictivos realizados por Thurmond et al (2005) indican que la pérdida fetal previa es el principal factor de aborto y muestran que el riesgo relativo de abortar es 2,5 veces mayor en las vacas con abortos previos. La explicación del por qué los abortos previos son uno de los mayores factores de riesgo estaría en parte dada por las metritis posteriores que dejarían un ambiente uterino dañado (Hanson et al., 2003) como también otros factores externos como los agentes de ciertas enfermedades infecciosas. Por ejemplo, las vacas previamente infectadas con Neospora caninum tendrían un mayor riesgo de repetir abortos (Thurmond y Hietala, 1996) y este podría ser en el caso de la cuenca lechera del Valle de Lerma un factor de aborto importante debido a la alta seroprevalencia de N. caninum hallada en los rodeos (Pereyra et al., 2020).

El mayor porcentaje observado de descartes y muertes/sacrificios en las vacas que abortaron coinciden con los resultados de Bartels et al. (2006) donde la probabilidad de sacrificio se incrementó 1,19 veces para las vacas con abortos. Del mismo modo Keshavarzi et al. (2020) estimaron en vacas que iniciaban una lactancia pos aborto una probabilidad 2,41 veces mayor de ser sacrificados debido a problemas reproductivos en comparación con las vacas que tienen partos normales. Otros estudios evidenciaron que las vacas que abortan tendrían 3,2 veces más riesgo de ser descartadas que aquellas que finalizan una gestación normal; sin embargo solo 1 de cada 6 era descartada a causa del aborto, con el riesgo de un nuevo aborto ya que éstas tendrían 5 veces más probabilidades de abortar posteriormente que las vacas sin antecedentes de abortos (Ghavi Hossein-Zadeh, 2013).
La información del presente trabajo ratifica y enfatiza la importancia de los abortos en las vacas lecheras, donde la ineficiencia y pérdidas de producción deben verse en forma integral ya que no solo se traduciría en la pérdida del ternero y de reemplazos de hembras sino también a todo lo relacionado al servicio y gestación, como los gastos de inseminación, tiempo, mano de obra, alimentación, etc. y lo relacionado a pérdidas productivas asociadas a un incremento en el intervalo entre partos, de secuelas como infertilidad, pérdidas embrionarias, descartes o muertes y aumentos del intervalo generacional en detrimento del progreso genético (Thurmond et al.,2005; Gädicke et al., 2010; Rafati et al., 2010).

Los presentes resultados señalan por un lado, efectos diferentes entre las vacas abortadas que continúan en lactancia y de aquellas que inician una nueva lactancia como ser: a) reducción en la producción de leche; b) aumento del intervalo entre partos; c) una mayor probabilidad de ocurrencia de eventos sanitarios reproductivos posteriores; d) una mayor probabilidad de descarte o muerte-sacrificio prematuros. Por otro lado, esta investigación muestra a partir de su importancia en la eficiencia y sustentabilidad de los tambos la necesidad de profundizar los estudios sobre las causas más importantes de los abortos, de las pérdidas embrionarias $\mathrm{y}$ de los partos prematuros e investigar las formas de prevenirlas.

\section{Agradecimientos.}

Los autores agradecen a los encargados y propietarios de los establecimientos lecheros por permitirnos acceder a sus registros y colaborar con el presente estudio.

\section{Referencias}

Bartels CJM, Van Schaik G, Veldhuisen JP, Van den Borne BHP, Wouda W, Dijkstra T. 2006. Effect of Neospora caninum serostatus on culling, reproductive performance and milk production in Dutch dairy herds with and without a history of Neospora caninum-associated abortion epidemics. Prev. Vet. Med. 77: 186-198.

Butler WR, Smith RD. 1989. Interrelationships between energy balance and postpartum reproductive function in dairy cattle. J. Dairy Sci. 72: 767-783.

Capuco AV, Akers RM, Smith JJ. 1997. Mammary growth in Holstein cows during the dry period: quantification of nucleic acids and histology. J. Dairy Sci. 80: 477-487.

Catalino MB, Casaro BG, Daglio MC. 2017 Cuantificación de pérdidas de gestación entre los días 35-100 en vacas de tambo de la Cuenca Mar y Sierras. Tesina para optar al grado de Veterinario, F.C. Veterinarias, UNCPBA, Tandil, 27 pp.

De Vries A. 2006. Economic value of pregnancy in dairy cattle. J. Dairy Sci. 89: 3876-3885. 
El-Tarabany MS. 2015. Impact of stillbirth and abortion on the subsequent fertility and productivity of Holstein, Brown Swiss and their crosses in subtropics. Trop. Anim. Health. Prod. 47: 1351-1356.

Fetrow J, Mcclary D, Harman R, Butcher K, Weaver L, Studer E, Ehrlich J, Etherington W, Guterbock W, Klingbord D, Reneau J, Williamson N. 1990. Calculating selected Reproductive Indices: Recommendations of the American Association of Bovine Practitioners. J. Dairy Sci. 73:78-90.

Forar A, Gay J, Hancock D. 1996. The frequency of edemic fetal loss in dairy cattle: a review. Theriogenology 43: 989-1000.

Fourichon C, Seegers H, Malher X. 2000. Effect of disease on reproduction in the dairy cow: a meta-analysis. Theriogenology 53: 1729-1759.

Gädicke P, Monti G. 2008. Aspectos epidemiológicos y de análisis del síndrome de aborto bovino. Arch. Med. Vet. 40: 223-234.

Gädicke P, Vidal R, Monti G. 2010. Economic effect of bovine abortion syndrome in commercial dairy herds in Southern Chile. Prev. Vet. Med. 97: 9-19.

Ghavi Hossein-Zadeh N. 2013. Effects of main reproductive and health problems on the performance of dairy cows: a review. Span. J. Agric. Res. 11 (3): 718-735.

Ghavi Hossein-Zadeh N, Ardalan M. 2011. Evaluation of the potential effects of abortion on the productive performance of Iranian Holstein dairy cows. Anim. Sci. J. 82: 117-121.

Gröhn Y, Erb H, Mc Culloch Ch, Saloniemi H. 1990. Epidemiology of reproductive disorders in dairy cattle: associations among host characteristics, disease and production. Prev. Vet. Med. 8: 25-39.

Hanson T, Bedrick EJ, Johnson WO, Thurmond MC. 2003. A mixture model for bovine abortion and foetal survival. Stat. Med. 22: 1725-1739.

Kaneene JB, Miller R. 1995. Risk factors for metritis in Michigan dairy cattle using herd- and cow-based modelling approaches. Prev. Vet. Med. 23: 183-200

Keshavarzi H, Sadeghi-Sefidmazgi A, Ghorbania GR, Kowsara R, Razmkabirb M, Amerc P. 2020. Effect of abortion on milk production, health, and reproductive performance of Holstein dairy cattle. Anim. Reprod. Sci. 217: 106458.

Lee JI, Kim IH. 2007 Pregnancy loss in dairy cows: the contributing factors, the effects on reproductive performance and the economic impact. J. Vet. Sci. 8: 283-288.

Melendez P, Pinedo P. 2007. The association between reproductive performance and milk yield in Chilean Holstein cattle. J. Dairy Sci. 90: 184-192

Miller R. 1986. Bovine Abortion. In: Morrow D (ed). Current Therapy In: Theriogenology. WB Saunders Company, Michigan State University, Michigan, USA.

Nigussie T. 2018. A Review on the Role of Energy Balance on Reproduction of Dairy Cow. J. Dairy Res. Tech. 1: 003.
Norman HD, Miller RH, Wright JR, Hutchison JL, Olson KM. 2012. Factors associated with frequency of abortions recorded through Dairy Herd Improvement test plans. J. Dairy Sci. 95: 4074-4084.

Pereyra WR, Suarez VH, Cardoso N, Gual I, Martínez GM, Capozzo AV, Mansilla, FC. 2020. Prevalencia sérica de Neospora caninum y factores de riesgo asociados a su transmisión en tambos de la provincia de Salta, Argentina. Rev. Argent. Microbiol. 53: 145-153.

Peter AT. 2000. Abortions in dairy cows: new insights and economic impact. Adv. Dairy Technol. 12: 233-244.

Plaizier JC, King GJ, Dekkers JC, Lissemore K. 1997. Estimation of economic values of indices for reproductive performance in dairy herds using computer simulation. J. Dairy Sci. 80: 27752783.

Rafati N, Mehrabani-Yeganeh, Hanson TE. 2010. Risk factors for abortion in dairy cows from commercial Holstein dairy herds in the Tehran region. Prev. Vet. Med. 96: 170-178.

Salasel B, Mokhtari A, Taktaz, T. 2010. Prevalence, risk factors for and impact of subclinical endometritis in repeat breeder dairy cows. Theriogenology 74: 1271-1278.

Suarez VH, Martínez GM. 2015. Características y Problemáticas Productivas - Sanitarias de la Lechería del Valle de Lerma (Salta). INTA Ediciones, Col. Investigación, desarrollo e innovación. 66 pp.

Thomsen PT, Houe H. 2006. Dairy cow mortality. A review. The Vet. quarterly 28: 122-129.

Thurmond MC, Hietala SK. 1996. Culling associated with Neospora caninum infection in dairy cows. Am. J. Vet. Res. 57: 1559-1562.

Thurmond MC, Picanso JP, Jameson CM. 1990. Considerations for use of descriptive epidemiology to investigate fetal loss in dairy cows. J. Am. Vet. Med. Assoc. 197: 1305-1312.

Thurmond MC, Branscum AJ, Johnson WO, Bedrick EJ, Hanson TE. 2005. Predicting the probability of abortion in dairy cows: $A$ hierarchical Bayesian logistic-survival model using sequential pregnancy data. Prev. Vet. Med. 68: 223-239.

Weaver LD, Goodger WJ. 1987. Design and economic evaluation of dairy reproductive hlth programs for large dairy herds. Comp. Contin. Educ. Pract. Vet. 9: 355-366.

Wilde CJ, Knight CH. 1998. Metabolic adaptations in mammary gland during the declining phase of lactation. J. Dairy Sci. 72: 1679-1692.

Wiltbank, MC, Baez GM, Garcia-Guerra A, Toledo MZ, Monteiro PL, Melo LF, Sartori R. 2016. Pivotal periods for pregnancy loss during the first trimester of gestation in lactating dairy cows. Theriogenology 86: 239-253. 